\title{
Responding to the need of the Society: Nepal Ambulance Service
}

\author{
Gongal R. , Vaidya P.
}

Emergency Medical Service (EMS) System is now considered a fundamental component of a health system of a society. This will be activated by a toll free three digit number which reaches a dispatch centre manned by trained Emergency Medical Dispatchers (EMDs) who will triage the calls, dispatch the nearest ambulance to the scene and also provide pre-arrival instructions to the caller. The ambulance with required facilities with trained paramedics - Emergency Medical Technicians (EMTs) ensure that key interventions are achieved and patients transported to the most appropriate facility. It is well accepted that timely intervention and rapid transport reduce morbidity and mortality.

However, in Nepal, the situation of EMS is virtually nonexistent. A study done in Patan Hospital in 2007 showed that only $10 \%$ of patients arriving to Emergencies came by ambulances, more than $50 \%$ came by taxis which included triage category I patients (who have life threatening conditions and need immediate help). But it is little solace for those who prefer the ambulance, as the study showed that only $17 \%$ of ambulances have oxygen and none had personnel who have had even basic first aid training. So the ambulance is a little more than a taxi with siren.

To respond to the need, Nepal Ambulance Service, a nonprofit non-governmental organization, was established. With the slogan "saving lives on the way", it aims to develop a system of professional ambulance service from scratch and spread it across the country, a system which is reliable, affordable and which runs twenty four hours seven days a week.

For the first time in the country, a toll free three digit number of 102 has been established for medical emergency for Kathmandu and Lalitpur which can be accessed by both Nepal Telecom landline and mobiles as well as Ncell. This connects to a dispatch centre manned by trained EMDs, where all calls are logged into a data base for further analysis for future improvement and directions. The EMDs mobilize one of the five nearest ambulance spread around the ring road of Kathmandu. The trained EMTs provide care en route to the hospital. NAS has also developed a network of another 12 ambulance providers which are asked to respond when NAS's own ambulances are busy. While these ambulances lack equipment and trained personnel meeting NAS standards, they ensure that NAS is able to dispatch an ambulance to all emergency 102 call.

The EMTs were trained by professionals from Stanford University Emergency Department. The Community Medical Assistant (CMAs) underwent three months training in Patan Hospital which included class room training, Wards, Emergency Room and Labour room based training. This was followed by rigorous examination. The graduates who were employed by NAS further underwent another three months of 'on the road training'.

After more than one and half year of service, NAS has provided service to more than 4000 patients. Twelve babies has been delivered successfully in the ambulances on the way to the hospital. NAS aims to expand its service to the whole of Kathmandu Valley and outside. It also recognizes the importance of partnering with the Government to take this to all parts of the country. Although there is still a long way to go, an EMS system is being built.

Call 102 for an ambulance and save lives 